\title{
HODOGRAPHIC STUDY OF NON-NEWTONIAN MHD ALIGNED STEADY PLANE FLUID FLOWS
}

\author{
P.V. NGUYEN and O.P. CHANDNA \\ Departmint of Mathematics and Statistics \\ University of Windsor \\ Windsor, Ontario N9B 3P4, Canada
}

(Received May 22, 1989)

\begin{abstract}
A study is made of non-Newtonian MHD aligned steady plane fluid flows to find exact solutions for various flow configurations. The equations of motion have been transformed to the hodograph plane. A Legendre-transform function is used to recast the equations in the hodograph plane in terms of this transform function. Solutions for various flow configurations are obtained. Applications are investigated for the fluids of finite and infinite electrical conductivity bringing out the similarities and contrasts in the solutions of these types of fluids. KEY WORDS AND PHRASES. Steady, Non-Newtonian Magnetofluid dynamic, incompressible, plane, Hodographic study, Aligned.

1980 AMS SUBJECT CLASSIFICATION CODE. 76 W05.
\end{abstract}

1. INTRODUCTION. Transformation techniques are often employed for solving non-linear partial differential equations and hodograph transformation method is one of these techniques which has been widely used in continuum mechanics. W. F. Ames [1] has given an excellent survey of this method together with its applications in various other fields. This paper deals with the application of this method for solving a system of non-linear partial differential equations guverning steady plane incompressible flow of an electrical conducting second-grade fluid in the presence of an aligned magnetic field. Recently, A. M. Siddiqui et al [2] used the hodograph and Legendre transformations to study non-Newtonian steady plane fluid flows. O. P. Chandna et al [3] has also applied this technique to Navier-Stokes equations. Since electrical conductivity is finite for most liquid metals and it is also finite for other electrically conducting second grade fluids to which single fluid model can be applied, our accounting for the finite electrical conductivity makes the flow problem realistic and attractive from both a physical and a mathematical point of view. We have also included electrically conducting second grade fluids of infinite electrical conductivity to make a thorough hodographic study of these fluid flows and to recognize the dawn and future of superconductivity in science.

We study our flows with the objective of obtaining exact solutions to various flow configurations. We start with reducing the order of governing equations by employing M. H. Martin's [4] perceptive idea of introducing vorticity and energy functions. The plan of this paper is as follow's: In section 2 the equations are cast into a convenient form for this work. Section 3 contains the transformation of equations to the hodograph plane so that the role of independent variables $x$. $y$ and the dependent variables $u, v$ (the two components of the velocity vector field) is interchanged. We introduce a Legendre-transform function of the streamfunction and recast all our equations in the hodograph plane in terms of this transform function in Section 4. Theoretical development 
of section 4 is illustrated by solutions to the following examples in section 5:

(a) flows with elliptic and circular streamlines

(b) hyperbolic flows

(c) spiral flows

(d) radial flows.

These applications are investigated for the fluids of finite and infinite electrical conductivity bringing out the similarity and contrasts in the solutions of these two types of fluids.

2. EQUATIONS OF MOTION. The steady, plane flow of an incompressible second-grade fluid of finite electrical conductivity is governed by the following system of equations:

$$
\begin{gathered}
\frac{\partial u}{\partial x}+\frac{\partial v}{\partial y}=0 \\
\rho\left(u \frac{\partial u}{\partial x}+v \frac{\partial u}{\partial y}\right)+\frac{\partial p}{\partial x} \\
=\mu\left(\frac{\partial^{2} u}{\partial x^{2}}+\frac{\partial^{2} u}{\partial y^{2}}\right)+\alpha_{1}\left[\frac { \partial } { \partial x } \left\{2 u \frac{\partial^{2} u}{\partial x^{2}}\right.\right. \\
\left.+2 v \frac{\partial^{2} u}{\partial x \partial y}+4\left(\frac{\partial u}{\partial x}\right)^{2}+2 \frac{\partial v}{\partial x}\left(\frac{\partial u}{\partial y}+\frac{\partial v}{\partial x}\right)\right\} \\
+\frac{\partial}{\partial y}\left\{\left(u \frac{\partial}{\partial x}+v \frac{\partial}{\partial y}\right)\left(\frac{\partial v}{\partial x}+\frac{\partial u}{\partial y}\right)\right. \\
\left.\left.+2 \frac{\partial u}{\partial x} \frac{\partial u}{\partial y}+2 \frac{\partial v}{\partial x} \frac{\partial v}{\partial y}\right\}\right] \\
+\alpha_{2}\left[\frac{\partial}{\partial x}\left\{4\left(\frac{\partial u}{\partial x}\right)^{2}+\left(\frac{\partial v}{\partial x}+\frac{\partial u}{\partial y}\right)^{2}\right\}\right] \\
-\mu^{*} H_{2}\left(\frac{\partial H_{2}}{\partial x}-\frac{\partial H_{1}}{\partial y}\right) \\
+\mu^{*} H_{1}\left(\frac{\partial H_{2}}{\partial x}-\frac{\partial H_{1}}{\partial y}\right) \\
\left.+v \frac{\partial v}{\partial x}\right)+\frac{\partial p}{\partial y} \\
+\mu\left(\frac{\partial^{2} v}{\partial x^{2}}+\frac{\partial^{2} v}{\partial y^{2}}\right)+\alpha_{1}\left[\frac { \partial } { \partial x } \left\{\left(u \frac{\partial}{\partial x}+v \frac{\partial}{\partial y}\right)\left(\frac{\partial v}{\partial x}+\frac{\partial u}{\partial y}\right)^{2}\right.\right. \\
\left.+2 \frac{\partial u}{\partial x} \frac{\partial u}{\partial y}+2 \frac{\partial v}{\partial x} \frac{\partial v}{\partial y}\right\}+\frac{\partial}{\partial y}\left\{2 u \frac{\partial^{2} v}{\partial x \partial y}+2 v \frac{\partial^{2} v}{\partial y^{2}}+4\left(\frac{\partial v}{\partial y}\right)^{2}\right. \\
\left.\left.+\frac{\partial u}{\partial y}\right)\right\}+K \\
\left.\left.+\frac{\partial}{\partial y}\left\{\frac{\partial H_{1}}{\partial y}\right)^{2}+\left(\frac{\partial u}{\partial y}+\frac{\partial v}{\partial x}\right)^{2}\right\}\right]
\end{gathered}
$$

where $u, v$ are the components of velocity field $\vec{V}, H_{1}, H_{2}$ the components of the magnetic vector field $\vec{H}$, and $p$ is the pressure function: all being functions of $x, y$. In this system $\rho, \mu, \mu^{*}, \sigma, \alpha_{1}$ and $\alpha_{2}$ are respectively the constant fluid density, the constant coefficient of viscosity. the con- 
stant magnetic permeability, the constant electrical conductivity and the normal stress moduli. Furthermore, $K$ is an arbitrary constant of integration obtained from the diffusion equation

$$
\operatorname{curl}\left[\vec{V} \times \vec{H}-\frac{1}{\mu^{*} \sigma} \operatorname{curl} \vec{H}\right]=\overrightarrow{0} \text {. }
$$

We now introduce the two dimensional vorticity function $\omega$, the current density function $j$ and energy function $e$ defined by

$$
\begin{gathered}
\omega=\frac{\partial v}{\partial x}-\frac{\partial u}{\partial y}, j=\frac{\partial H_{2}}{\partial x}-\frac{\partial H_{1}}{\partial y} \\
e=\frac{1}{2} \rho q^{2}-\alpha_{1}\left(u \nabla^{2} u+v \nabla^{2} v\right)-\frac{1}{4}\left(3 \alpha_{1}+2 \alpha_{2}\right)\left|\tilde{A}_{1}\right|^{2}+p .
\end{gathered}
$$

where $q^{2}=u^{2}+v^{2}, \nabla^{2}=\frac{\partial^{2}}{\partial x^{2}}+\frac{\partial^{2}}{\partial y^{2}}$ and

$$
\left|\tilde{A}_{1}\right|^{2}=4\left(\frac{\partial u}{\partial x}\right)^{2}+4\left(\frac{\partial v}{\partial y}\right)^{2}+\alpha\left(\frac{\partial u}{\partial y}+\frac{\partial v}{\partial x}\right)^{2}
$$

into the above system of equations and obtain the following system:

$$
\begin{array}{cc}
\frac{\partial u}{\partial x}+\frac{\partial v}{\partial y}=0 & \text { (continuity) } \\
\left.\begin{array}{rl}
\frac{\partial e}{\partial x}=\rho v \omega-\mu \frac{\partial \omega}{\partial y}-\alpha_{1} v \nabla^{2} \omega-\mu^{*} j H_{2} \\
\frac{\partial e}{\partial y}=-\rho u \omega+\mu \frac{\partial \omega}{\partial x}+\alpha_{1} u \nabla^{2} \omega+\mu^{*} j H_{1}
\end{array}\right\} & \text { (linear momentum) } \\
u H_{2}-v H_{1}=\frac{1}{\mu^{*} \sigma} j+K & \text { (diffusion) } \\
\frac{\partial H_{1}}{\partial x}+\frac{\partial H_{2}}{\partial y}=0 & \text { (solenoidal) } \\
\frac{\partial H_{2}}{\partial x}-\frac{\partial H_{1}}{\partial y}=j & \text { (current density) } \\
\frac{\partial v}{\partial x}-\frac{\partial u}{\partial y}=\omega & \text { (vorticity) }
\end{array}
$$

of seven partial differential equations in seven unknown functions $u, v, \omega, H_{1}, H_{2}, j$ and $e$ as functions of $x, y$. This system governs the motion of second-grade fluid of finite electrical conductivity. For the motion of second-grade fluid of infinite electrical conductivity, we only replace the diffusion equation in the above system by $u H_{2}-v H_{1}=K$.

ALIGNED FLOW. A flow is said to be an aligned or parallel flow if the velocity and the magnetic fields are everywhere parallel. Taking our flow to be an aligned flow, there exists some scalar function $f(x, y)$, called the proportionality function, such that

$$
\vec{H}=f(x, y) \vec{V}
$$

Introducing this definition of the magnetic vector field in the above system, the aligned flow is governed by the following system of seven equations

$$
\begin{gathered}
\frac{\partial u}{\partial x}+\frac{\partial v}{\partial y}=0 \\
\frac{\partial e}{\partial x}=\rho v \omega-\mu \frac{\partial \omega}{\partial y}-\alpha_{1} v \nabla^{2} \omega-\mu^{*} f v j \\
\frac{\partial e}{\partial y}=-\rho u \omega+\mu \frac{\partial \omega}{\partial x}+\alpha_{1} u \nabla^{2} \omega+\mu^{*} f u j
\end{gathered}
$$




$$
\begin{gathered}
\frac{1}{\mu^{*} \sigma} j+K=0 \\
u \frac{\partial f}{\partial x}+v \frac{\partial f}{\partial y}=0 \\
f \omega+v \frac{\partial f}{\partial x}-u \frac{\partial f}{\partial y}=j \\
\frac{\partial v}{\partial x}-\frac{\partial u}{\partial y}=\omega
\end{gathered}
$$

is six unknown functions $u(x, y), v(x, y), \omega(x, y), f(x, y), j(x, y), e(x, y)$ and an arbitrary constant $K$. Once a solution of this system is determined, the pressure and the magnetic functions are obtained by using the definition of $e$ in (1) and the definition of $\vec{H}$ in (3) respectively.

3. EQUATIONS IN THE HODOGRAPH PLANE. Letting the flow variables $u(x, y), v(x, y)$ be such that, in the region of flow, the Jacobian

$$
J(x, y)=\frac{\partial(u, v)}{\partial(x, y)} \neq 0, \quad 0<|J|<\infty
$$

we may consider $x$ and $y$ as functions of $u$ and $v$. By means of $x=x(u, v), y=y(u, v)$, we derive the following relations

and

$$
\left.\begin{array}{ll}
\frac{\partial u}{\partial x}=J \frac{\partial y}{\partial v}, & \frac{\partial u}{\partial y}=-J \frac{\partial x}{\partial v} \\
\frac{\partial v}{\partial x}=-J \frac{\partial y}{\partial u}, & \frac{\partial v}{\partial y}=J \frac{\partial x}{\partial u}
\end{array}\right\}
$$

$$
\left.\begin{array}{l}
\frac{\partial g}{\partial x}=\frac{\partial(g, y)}{\partial(x, y)}=\bar{J} \frac{\partial(\bar{g}, y)}{\partial(u, v)} \\
\frac{\partial g}{\partial y}=-\frac{\partial(g, x)}{\partial(x, y)}=\bar{J} \frac{\partial(x, \bar{g})}{\partial(u, v)}
\end{array}\right\}
$$

where $g=g(x, y)=g(x(u, v), y(u, v))=\bar{g}(u, v)$ is any continuously differentiable function and

$$
J=J(x, y)=\frac{\partial(u, v)}{\partial(x, y)}=\left[\frac{\partial(x, y)}{\partial(u, v)}\right]^{-1}=\bar{J}(u, v) .
$$

Employing these transformation relations for the first order partial derivatives appearing in system of equations (4) - (10) and the transformation equations for the functions $\omega, j, f, e$ defined by

$$
\begin{aligned}
\omega(x, y) & =\omega(x(u, v), y(u, v))=\bar{\omega}(u, v), \\
j(x, y)=j(x(u, v), y(u, v)) & =\bar{j}(u, v), \\
f(x, y)=f(x(u, v), y(u, v)) & =\bar{f}(u, v), \\
e(x, y)=e(x(u, v), y(u, v)) & =\bar{e}(u, v),
\end{aligned}
$$

the system (4)-(10) is transformed into the following system of seven equations in the $(u, v)$-plane:

$$
\begin{gathered}
\frac{\partial x}{\partial u}+\frac{\partial y}{\partial v}=0 \\
\bar{J} \frac{\partial(\bar{e}, y)}{\partial(u, v)}=\rho v \bar{\omega}-\mu \bar{J} W_{1}-\alpha_{1} v \bar{J}\left[\frac{\partial\left(x, \bar{J} W_{1}\right)}{\partial(u, v)}+\frac{\partial\left(\bar{J} W_{2}, y\right)}{\partial(u, v)}\right]-\mu^{*} \bar{f} v \bar{j} \\
\bar{J} \frac{\partial(x, \bar{e})}{\partial(u, v)}=-\rho u \bar{\omega}+\mu \bar{J} W_{2}+\alpha_{1} u \bar{J}\left[\frac{\partial\left(x, \bar{J} W_{1}\right)}{\partial(u, v)}+\frac{\partial\left(\bar{J} W_{2}, y\right)}{\partial(u, v)}\right]+\mu^{*} \bar{f} u \bar{j}
\end{gathered}
$$




$$
\begin{gathered}
\frac{1}{\mu^{*} \sigma} \bar{j}+K=0 \\
u \frac{\partial(\bar{f}, y)}{\partial(u, v)}+v \frac{\partial(x, \bar{f})}{\partial(u, v)}=0 \\
\bar{f} \bar{\omega}+\bar{J}\left[v \frac{\partial(\bar{f}, y)}{\partial(u, v)}-u \frac{\partial(x, \bar{f})}{\partial(u, v)}\right]=\bar{j} \\
\bar{J}\left(\frac{\partial x}{\partial v}-\frac{\partial y}{\partial u}\right)=\bar{\omega}
\end{gathered}
$$

where

$$
\left.\begin{array}{l}
W_{1}=W_{1}(u, v)=\frac{\partial(x, \bar{\omega})}{\partial(u, v)} \\
W_{2}=W_{2}(u, v)=\frac{\partial(\bar{\omega}, y)}{\partial(u, v)}
\end{array}\right\}
$$

for the six unknown functions, $x, y, \bar{\omega}, \bar{e}, \bar{j}, \bar{f}$ of $u, v$ and an arbitrary constant $K$ when $\bar{J}, W_{1}$, $W_{2}$ are eliminated, using (14) and (22).

Once a solution $x=x(u, v), y=y(u, v), \bar{\omega}=\bar{\omega}(u, v), \bar{e}=\bar{e}(u, v), \bar{j}=\bar{j}(u, v), \bar{f}=$ $\bar{f}(u, v)$ is determined, we are led to the solution of $u=u(x, y), v=v(x, y)$ and therefore $\omega=$ $\bar{\omega}(u(x, y), v(x, y))=\omega(x, y), e=e(x, y), j=j(x, y), f=f(x, y)$ for the system (4) - (10) governing the finitely conducting flow.

The above analysis also holds true for infinitely conducting second-grade fluid flows. However, for these flows, the arbitrary constant $K=0$ and equation (7) and its transformed equation (18) are identically satisfied.

4. EQUATIONS FOR THE LEGENDRE TRANSFORM FUNCTION AND $\bar{F}(U, V)$. The equation of continuity implies the existence of a streamfunction $\psi(x, y)$ such that

$$
d \psi=-v d x+u d y \quad \text { or } \quad \frac{\partial \psi}{\partial x}=-v, \quad \frac{\partial \psi}{\partial y}=u
$$

Likewise, (15) implies the existence of a function $L(u, v)$, called the Legendre transform function of the streamfunction $\psi(x, y)$, so that

$$
d L=-y d u+x d v \quad \text { or } \quad \frac{\partial L}{\partial u}=-y, \quad \frac{\partial L}{\partial v}=x
$$

and the two functions $\psi(x, y), L(u, v)$ are related by

$$
L(u, v)=v x-u y+\psi(x, y) .
$$

Introducing $L(u, v)$ into the system (15)-(21), with $\bar{J}, W_{1}, W_{2}$ given by (14), (22) respectively, it follows that (15) is identically satisfied and this system may be replaced by

$$
\begin{gathered}
\bar{J} \frac{\partial\left(\frac{\partial L}{\partial u}, \bar{e}\right)}{\partial(u, v)}=\rho v \bar{\omega}-\mu \bar{J} W_{1}-\alpha_{1} v \bar{J}\left[\frac{\partial\left(\frac{\partial L}{\partial v}, \bar{J} W_{1}\right)}{\partial(u, v)}+\frac{\partial\left(\frac{\partial L}{\partial u}, \bar{J} W_{2}\right)}{\partial(u, v)}\right]-\mu^{*} \bar{f} v \bar{j} \\
\bar{J} \frac{\partial\left(\frac{\partial L}{\partial v}, \bar{e}\right)}{\partial(u, v)}=-\rho u \bar{\omega}+\mu \bar{J} W_{2}+\alpha_{1} u \bar{J}\left[\frac{\partial\left(\frac{\partial L}{\partial v}, \bar{J} W_{1}\right)}{\partial(u, v)}+\frac{\partial\left(\frac{\partial L}{\partial u}, \bar{J} W_{2}\right)}{\partial(u, v)}\right]+\mu^{*} \bar{f} u \bar{j} \\
\frac{1}{\mu^{*} \sigma} \bar{j}+K=0 \\
u \frac{\partial\left(\frac{\partial L}{\partial u}, \bar{f}\right)}{\partial(u, v)}+v \frac{\partial\left(\frac{\partial L}{\partial v}, \bar{f}\right)}{\partial(u, v)}=0
\end{gathered}
$$




$$
\begin{gathered}
\bar{f} \bar{\omega}+\bar{J}\left[v \frac{\partial\left(\frac{\partial L}{\partial u}, \bar{f}\right)}{\partial(u, v)}-u \frac{\partial\left(\frac{\partial L}{\partial v}, \bar{f}\right)}{\partial(u, v)}\right]=\bar{j} \\
\bar{J}\left(\frac{\partial^{2} L}{\partial v^{2}}+\frac{\partial^{2} L}{\partial u^{2}}\right)=\bar{\omega}
\end{gathered}
$$

where now

$$
\begin{gathered}
\bar{J}=\left[\frac{\partial^{2} L}{\partial u^{2}} \frac{\partial^{2} L}{\partial v^{2}}-\left(\frac{\partial^{2} L}{\partial u \partial v}\right)^{2}\right]^{-1} \\
W_{1}=\frac{\partial\left(\frac{\partial L}{\partial v}, \bar{\omega}\right)}{\partial(u, v)}, \quad W_{2}=\frac{\partial\left(\frac{\partial L}{\partial u}, \bar{\omega}\right)}{\partial(u, v)}
\end{gathered}
$$

for the five functions $L(u, v), \bar{\omega}(u, v), \bar{e}(u, v), \bar{j}(u, v), \bar{f}(u, v)$ and an arbitrary constant $K$, after $\bar{J}, W_{1}, W_{2}$ are eliminated.

By using the integrability condition

$$
\left(\bar{J} \frac{\partial^{2} L}{\partial u \partial v} \frac{\partial}{\partial v}-\bar{J} \frac{\partial^{2} L}{\partial v^{2}} \frac{\partial}{\partial u}\right)\left[\bar{J} \frac{\partial\left(\frac{\partial L}{\partial u}, \bar{e}\right)}{\partial(u, v)}\right]=\left(\bar{J} \frac{\partial^{2} L}{\partial u^{2}} \frac{\partial}{\partial v}-\bar{J} \frac{\partial^{2} L}{\partial u \partial v} \frac{\partial}{\partial u}\right)\left[\bar{J} \frac{\partial\left(\frac{\partial L}{\partial v}, \bar{e}\right)}{\partial(u, v)}\right]
$$

i.e. $\frac{\partial^{2} e}{\partial x \partial y}=\frac{\partial^{2} e}{\partial y \partial x}$ in $(x, y)$-plane, we eliminate $\bar{e}(u, v)$ from (26) and (27) and obtain

$$
\begin{aligned}
& \mu\left[\frac{\partial\left(\frac{\partial L}{\partial v}, \bar{J} W_{1}\right)}{\partial(u, v)}+\frac{\partial\left(\frac{\partial L}{\partial u}, \bar{J} W_{2}\right)}{\partial(u, v)}\right] \\
& +\alpha_{1}\left[v \frac{\partial\left(\frac{\partial L}{\partial v}, \bar{J}\left\{\partial\left(\frac{\partial L}{\partial v}, \bar{J} W_{1}\right) / \partial(u, v)+\partial\left(\frac{\partial L}{\partial u}, \bar{J} W_{2}\right) / \partial(u, v)\right\}\right)}{\partial(u, v)}\right. \\
& \left.\quad+u \frac{\partial\left(\frac{\partial L}{\partial u}, \bar{J}\left\{\partial\left(\frac{\partial L}{\partial v}, \bar{J} W_{1}\right) / \partial(u, v)+\partial\left(\frac{\partial L}{\partial u}, \bar{J} W_{2}\right) / \partial(u, v)\right\}\right)}{\partial(u, v)}\right] \\
& \quad+\mu^{*} \bar{f}\left[v \frac{\partial\left(\frac{\partial L}{\partial v}, \bar{j}\right)}{\partial(u, v)}+u \frac{\partial\left(\frac{\partial L}{\partial u}, \bar{j}\right)}{\partial(u, v)}\right]=\rho\left(u W_{2}+v W_{1}\right)
\end{aligned}
$$

(a) Since $\bar{j}$ has a constant value $-\mu^{*} \sigma K$ for a finitely conducting fluid as given by (28), it follows that $L(u, v), \bar{f}(u, v)$ satisfy equations $(29),(30)$ and

$$
\begin{aligned}
& \mu\left[\frac{\partial\left(\frac{\partial L}{\partial v}, \bar{J} W_{1}\right)}{\partial(u, v)}+\frac{\partial\left(\frac{\partial L}{\partial u}, \bar{J} W_{2}\right)}{\partial(u, v)}\right] \\
& \quad+\alpha_{1}\left[v \frac{\partial\left(\frac{\partial L}{\partial v}, \bar{J}\left\{\partial\left(\frac{\partial L}{\partial v}, \bar{J} W_{1}\right) / \partial(u, v)+\partial\left(\frac{\partial L}{\partial u}, \bar{J} W_{2}\right) / \partial(u, v)\right\}\right)}{\partial(u, v)}\right. \\
& \left.\quad+u \frac{\partial\left(\frac{\partial L}{\partial u}, \bar{J}\left\{\partial\left(\frac{\partial L}{\partial v}, \bar{J} W_{1}\right) / \partial(u, v)+\partial\left(\frac{\partial L}{\partial u}, \bar{J} W_{2}\right) / \partial(u, v)\right\}\right)}{\partial(u, v)}\right] \\
& \quad=\rho\left(u W_{2}+v W_{1}\right) .
\end{aligned}
$$

(b) Equation (28) is identically satisfied for an infinitely conducting fluid flow and $\bar{j}$ is given by (30). Eliminating $\bar{j}$ from (30) and (34), we find that for these flows $L(u, v)$ and $\bar{f}(u, v)$ satisfy equations (29) and 


$$
\begin{aligned}
\left(\mu^{*} \bar{f}^{2}\right. & -\rho)\left(u W_{1}+v W_{2}\right)+\mu\left[\frac{\partial\left(\frac{\partial L}{\partial v}, \bar{J} W_{1}\right)}{\partial(u, v)}+\frac{\partial\left(\frac{\partial L}{\partial u}, \bar{J} W_{2}\right)}{\partial(u, v)}\right] \\
& +\alpha_{1}\left[v \frac{\partial\left(\frac{\partial L}{\partial v}, \bar{J}\left\{\partial\left(\frac{\partial L}{\partial v}, \bar{J} W_{1}\right) / \partial(u, v)+\partial\left(\frac{\partial L}{\partial u}, \bar{J} W_{2}\right) / \partial(u, v)\right\}\right)}{\partial(u, v)}\right. \\
& \left.+u \frac{\partial\left(\frac{\partial L}{\partial u}, \bar{J}\left\{\partial\left(\frac{\partial L}{\partial v}, \bar{J} W_{1}\right) / \partial(u, v)+\partial\left(\frac{\partial L}{\partial u}, \bar{J} W_{2}\right) / \partial(u, v)\right\}\right)}{\partial(u, v)}\right] \\
& +\mu^{*} \bar{f}^{2}\left[\frac{\partial\left(\frac{\partial L}{\partial v}, \bar{J} F_{1}\right)}{\partial(u, v)}-\frac{\partial\left(\frac{\partial L}{\partial u}, \bar{J} F_{2}\right)}{\partial(u, v)}\right] \\
& +2 \mu^{*} \bar{f}\left[v \frac{\partial \bar{f}}{\partial u}-u \frac{\partial \bar{f}}{\partial v}\right]=0
\end{aligned}
$$

where $F_{1}$ and $F_{2}$ are defined as

$$
F_{1}=F_{1}(u, v)=\frac{\partial\left(\frac{\partial L}{\partial u}, \bar{f}\right)}{\partial(u, v)}, \quad F_{2}=F_{2}(u, v)=\frac{\partial\left(\frac{\partial L}{\partial v}, \bar{f}\right)}{\partial(u, v)} .
$$

Summing up, we have the following theorems for finitely conducting and infinitely conducting fluid flows:

THEOREM I. If $L(u, v)$ is the Legendre transform function of a streamfunction of steady plane aligned flow of an incompressible second-grade fluid of finite electrical conductivity and $\bar{f}(u, v)$ is the transformed proportionality function, then $L(u, v)$ and $\bar{f}(u, v)$ must satisfy equations (29), (30) and (35) where $\bar{j}(u, v), \bar{\omega}(u, v), \bar{J}(u, v), W_{1}(u, v), W_{2}(u, v)$ are given by equations (28), (31) to (33).

THEOREM II. If $L(u, v)$ is the Legendre transform function of a streamfunction of the equations governing steady plane aligned flow of an incompressible second-grade fluid of infinite electrical conductivity and $\bar{f}(u, v)$ is the transformed proportionality function, then $L(u, v)$ and $\bar{f}(u, v)$ must satisfy equations (29) and (36) where $\bar{j}(u, v), \bar{\omega}(u, v), \bar{J}(u, v), W_{1}(u, v), W_{2}(u, v), F_{1}(u, v)$, $F_{2}(u, v)$ are given by (30) to (33) and (37).

Once a solution $L=L(u, v), \bar{f}=\bar{f}(u, v)$ is found, for which $\bar{J}$ evaluated from (32) satisfy $0<|J|<\infty$, the solutions for the velocity components are obtained by solving equations (24) simultaneously. Having obtained the velocity components $u=u(x, y), v=v(x, y)$, we obtain $f(x, y)$ in the physical plane from the solution for $\bar{f}(u, v)$ in the hodograph plane. We, then, obtain the vorticity, the current density and the energy functions by using $\vec{V}(x, y)$ and $f(x, y)$ in equations (10), (9), (5) and (6). Finally, the pressure function and the magnetic vector ficld are determined from (1) and (3).

As various assumed forms for Legendre transform function are best handled if polar coordinates in the hodograph plane are employed, we now develop the results of the above theorems in polar coordinates $(q, \theta)$ in the hodograph plane. Expressing

$$
u+i v=q e^{i \theta}
$$

we have the following transformations: 


$$
\begin{gathered}
\frac{\partial}{\partial u}=\cos \theta \frac{\partial}{\partial q}-\frac{\sin \theta}{q} \frac{\partial}{\partial \theta}, \quad \frac{\partial}{\partial v}=\sin \theta \frac{\partial}{\partial q}+\frac{\cos \theta}{q} \frac{\partial}{\partial \theta} \\
\frac{\partial(F, G)}{\partial(u, v)}=\frac{\partial\left(F^{*}, G^{*}\right)}{\partial(q, \theta)} \cdot \frac{\partial(q, \theta)}{\partial(u, v)}=\frac{1}{q} \frac{\partial\left(F^{*}, G^{*}\right)}{\partial(q, \theta)}
\end{gathered}
$$

where $F(u, v)=F^{*}(q, \theta), G(u, v)=G^{*}(q, \theta)$ are continuously differentiable functions. On using these relations, and regarding $(q, \theta)$ as new independent variables, the expressions for $\bar{J}, \bar{\omega}, W_{1}$. $W_{2}, F_{1}, F_{2}$ and $\bar{j}$ in the $(q, \theta)$ plane, take the forms

$$
\begin{aligned}
& J^{*}(q, \theta)=q^{4}\left[q^{2} \frac{\partial^{2} L^{*}}{\partial q^{2}}\left(q \frac{\partial L^{*}}{\partial q}+\frac{\partial^{2} L^{*}}{\partial \theta^{2}}\right)-\left(\frac{\partial L^{*}}{\partial \theta}-q \frac{\partial^{2} L^{*}}{\partial q \partial \theta}\right)^{2}\right]^{-1} \\
& \omega^{*}(q, \theta)=J^{*}\left[\frac{\partial^{2} L}{\partial q^{2}}+\frac{1}{q^{2}} \frac{\partial^{2} L^{*}}{\partial \theta^{2}}+\frac{1}{q} \frac{\partial L^{*}}{\partial q}\right] \\
& W_{1}^{*}(q, \theta)=\frac{1}{q} \frac{\partial\left(\sin \theta \frac{\partial L^{*}}{\partial q}+\frac{\cos \theta}{q} \frac{\partial L^{*}}{\partial \theta}, \omega^{*}\right)}{\partial(q, \theta)} \\
& W_{2}^{*}(q, \theta)=\frac{1}{q} \cdot \frac{\partial\left(\cos \theta \frac{\partial L^{*}}{\partial q}-\frac{\sin \theta}{q} \frac{\partial L^{*}}{\partial \theta}, \omega^{*}\right)}{\partial(q, \theta)} \\
& F_{1}^{*}(q, \theta)=\frac{1}{q} \frac{\partial\left(\cos \theta \frac{\partial L^{*}}{\partial q}-\frac{\sin \theta}{q} \frac{\partial L^{*}}{\partial \theta}, f^{*}\right)}{\partial(q, \theta)} \\
& F_{2}^{*}(q, \theta)=\frac{1}{q} \frac{\partial\left(\sin \theta \frac{\partial L^{*}}{\partial q}+\frac{\cos \theta}{q} \frac{\partial L^{*}}{\partial \theta}, f^{*}\right)}{\partial(q, \theta)} \\
& j^{*}(q, \theta)=f^{*} \omega^{*}+J^{*}\left[\sin \theta \frac{\partial\left(\cos \theta \frac{\partial L^{*}}{\partial q}-\frac{\sin \theta}{q} \frac{\partial L^{*}}{\partial \theta}, f^{*}\right)}{\partial(q, \theta)}\right. \\
&\left.-\cos \theta \frac{\partial\left(\sin \theta \frac{\partial L^{*}}{\partial q}+\frac{\cos \theta}{q} \frac{\partial L^{*}}{\partial \theta}, f^{*}\right)}{\partial(q, \theta)}\right]
\end{aligned}
$$

$j^{*}(q, \theta)=-\mu^{*} \sigma K$ is taken in (46) if the fluid is finitely conducting. Equations (29), (35) and (36) are transformed to the $(q, \theta)$-plane as

$$
\begin{gathered}
\frac{\partial^{2} L^{*}}{\partial q^{2}} \frac{\partial f^{*}}{\partial \theta}-\frac{\partial^{2} L^{*}}{\partial q \partial \theta} \frac{\partial f^{*}}{\partial q}+\frac{1}{q} \frac{\partial L^{*}}{\partial \theta} \frac{\partial f^{*}}{\partial q}=0 \\
\mu \chi^{*}+\alpha_{1}\left[\sin \theta \frac{\partial\left(\sin \theta \frac{\partial L^{*}}{\partial q}+\frac{\cos \theta}{q} \frac{\partial L^{*}}{\partial \theta}, J^{*} \chi^{*}\right)}{\partial(q, \theta)}\right] \\
\left.+\cos \theta \frac{\partial\left(\cos \theta \frac{\partial L^{*}}{\partial q}-\frac{\sin \theta}{q} \frac{\partial L^{*}}{\partial \theta}, J^{*} \chi^{*}\right)}{\partial(q, \theta)}\right] \\
=\rho q\left(\cos \theta W_{2}^{*}+\sin \theta W_{1}^{*}\right)
\end{gathered}
$$




$$
\begin{aligned}
\mu \chi^{*}+ & \alpha_{1}\left[\sin \theta \frac{\partial\left(\sin \theta \frac{\partial L^{*}}{\partial q}+\frac{\cos \theta}{q} \frac{\partial L^{*}}{\partial \theta}, J^{*} \chi^{*}\right)}{\partial(q, \theta)}\right. \\
& \left.+\cos \theta \frac{\partial\left(\cos \theta \frac{\partial L^{*}}{\partial q}-\frac{\sin \theta}{q} \frac{\partial L^{*}}{\partial \theta}, J^{*} \chi^{*}\right)}{\partial(q, \theta)}\right] \\
& +\mu^{*} f^{*} q\left[\frac{\partial\left(\sin \theta \frac{\partial L^{*}}{\partial q}+\frac{\cos \theta}{q} \frac{\partial L^{*}}{\partial \theta}, J^{*} F_{1}^{*}\right)}{\partial(q, \theta)}\right. \\
& \left.-\frac{\partial\left(\cos \theta \frac{\partial L^{*}}{\partial q}-\frac{\sin \theta}{q} \frac{\partial L^{*}}{\partial \theta}, J^{*} F_{2}^{*}\right)}{\partial(q, \theta)}\right]-2 \mu^{*} f^{*} \frac{\partial f^{*}}{\partial \theta} \\
& =\rho q\left(\cos \theta W_{2}^{*}+\sin \theta W_{1}^{*}\right)
\end{aligned}
$$

where $\chi^{*}$ is defined as

$$
\begin{aligned}
\chi^{*}=\chi^{*}(q, \theta)= & \frac{1}{q}\left\{\frac{\partial\left(\sin \theta \frac{\partial L^{*}}{\partial q}+\frac{\cos \theta}{q} \frac{\partial L^{*}}{\partial \theta}, J^{*} W_{1}^{*}\right)}{\partial(q, \theta)}\right. \\
& \left.+\frac{\partial\left(\cos \theta \frac{\partial L^{*}}{\partial q}-\frac{\sin \theta}{q} \frac{\partial L^{*}}{\partial \theta}, J^{*} W_{2}^{*}\right)}{\partial(q, \theta)}\right\}
\end{aligned}
$$

Having developed the above transformations, we state the following corollaries which respectively follow from theorem I and II:

COROLLARY I. If $L^{*}(q, \theta)$ and $f^{*}(q, \theta)$ is the Legendre transform function of a streamfunction and the proportionality function respectively of the equations governing the motion of steady plane aligned flow of an incompressible second-grade fluid of finite electrical conductivity, then $L^{*}(q, \theta)$ and $f^{*}(q, \theta)$ must satisfy equations (46), (47) and (48) where $J^{*}(q, \theta), \omega^{*}(q, \theta), W_{1}^{*}(q, \theta), W_{2}^{*}(q, \theta)$ and $\chi^{*}(q, \theta)$ are given by $(40)$ to $(43)$ and $(50)$.

COROLlARY II. If $L^{*}(q, \theta)$ and $f^{*}(q, \theta)$ are the Legendre transform of a streamfunction and the proportionality function of the equations governing the motion of steady plane aligned flow of an incompressible second-grade fluid of infinite electrical conductivity, then $L^{*}(q, \theta)$ and $f^{*}(q, \theta)$ must satisfy equations (47) and (49) where $J^{*}(q, \theta), \omega^{*}(q, \theta), W_{1}^{*}(q, \theta), W_{2}^{*}(q, \theta), F_{1}^{*}(q, \theta), F_{2}^{*}(q, \theta)$ and $\chi^{*}(q, \theta)$ are given by (40) to (45) and (50).

Once a solution $L^{*}(q, \theta), f^{*}(q, \theta)$ is obtained, we employ the relations

$$
x=\sin \theta \frac{\partial L^{*}}{\partial q}+\frac{\cos \theta}{q} \frac{\partial L^{*}}{\partial \theta}, \quad y=\frac{\sin \theta}{q} \frac{\partial L^{*}}{\partial \theta}+\cos \theta \frac{\partial L^{*}}{\partial q}
$$

and (38) to obtain the velocity components $u=u(x, y), v=v(x, y)$ in the physical plane. Following the determination of velocity components $u+i v=q e^{i \theta}$ in physical plane we get $f(x, y)$ and the other remaining flow variables. 
5. APPLICATIONS. In this section we investigate various problems as applications of Theorem I and II, and their corollaries.

APPLICATION I. Let

$$
L(u, v)=A u^{2}+B v^{2}+C u+D v+E
$$

be the Legendre transform function such that $A, B, C, D, E$ are arbitrary constants and $A, B$ are nonzero. Using (52) in equations (31) to (33), we get

$$
\bar{J}=\frac{1}{4 A B}, \quad \bar{\omega}=\frac{A+B}{2 A B}, \quad W_{1}=0, \quad W_{2}=0 .
$$

We now consider finitely conducting and infinitely conducting cases separately by applying theorem I and theorem II respectively.

FINITELY CONDUCTING FLUID. Eliminating $L(u, v), \quad \bar{\omega}(u, v), \quad \bar{J}(u, v), \quad W_{1}(u, v), \quad W_{2}(u, v)$ and $\bar{j}(u, v)$ from equations (29), (30), (35) by using the expressions for these functions from $(52),(53)$ and (28), we find that equation (35) is identically satisfied and $\bar{f}(u, v)$ must satisfy

$$
\left.\begin{array}{r}
A u \frac{\partial \bar{f}}{\partial v}-B v \frac{\partial \bar{f}}{\partial u}=0 \\
(A+B) \bar{f}+A v \frac{\partial \bar{f}}{\partial v}+B u \frac{\partial \bar{f}}{\partial u}+2 A B K \mu^{*} \sigma=0
\end{array}\right\}
$$

if $L(u, v)$ given by $(52)$ is the Legendre transform function of a streamfunction of finitely conducting fluid flow.

Solving equations (54), we have

$$
\bar{f}(u, v)=\left\{\begin{array}{cc}
\left(u^{2}+v^{2}\right)^{-\frac{1}{2}-\frac{B}{2 \Lambda}} \phi(u)-\frac{2 A B}{A+B} K \mu^{*} \sigma ; & A \neq-B \\
A K \mu^{*} \sigma \ln \left(u^{2}+v^{2}\right)+\psi(u) ; & A=-B
\end{array}\right\}
$$

where arbitrary functions $\phi(u)$ and $\psi(u)$ must satisfy

$$
\left\{B \phi^{\prime}(u)\right\} v^{2}+\left\{B \phi^{\prime}(u) u^{2}+\frac{1}{A}\left(A^{2}-B^{2}\right) u \phi(u)\right\}=0
$$

and

$$
\left\{\psi^{\prime}(u)\right\} v^{3}+\left\{4 A K \mu^{*} \sigma u\right\} v+\left\{u^{2} \psi^{\prime}(u)\right\}=0
$$

Since equations (56) and (57) hold true for every $v$, and $A \neq 0, B \neq 0$, it follows that we have the following three possible cases:

(i) $L(u, v)=A\left(u^{2}+v^{2}\right)+C u+D v+E, \quad \bar{f}(u, v)=C_{1}\left(u^{2}+v^{2}\right)^{-1}-A K \mu^{*} \sigma$ when $A=B \neq 0$ and $C$ is an arbitrary constant.

(ii) $\quad L(u, v)=A u^{2}+B v^{2}+C u+D v+E, \quad \bar{f}(u, v)=\frac{-2 . A B K^{\circ} \mu^{\circ} \sigma}{A+B}$ when $A \neq 0, \quad B \neq 0$ and $A \neq \pm B$.

(iii) $L(u, v)=A\left(u^{2}-v^{2}\right)+C u+D v+E, \quad \bar{f}(u, v)=C_{2} \quad$ when $\quad A=-B \neq 0, \quad C_{2}$ is an arbitrary constant and $K=0$.

We now proceed to study these three cases separately.

CASE (I). Using $L(u, v)=A\left(u^{2}+v^{2}\right)+C u+D v+E$ in (24) and solving the resulting equations simultaneously, we get

$$
u(x, y)=-\left(\frac{y+C}{2 A}\right), \quad v(x, y)=\left(\frac{x-D}{2 A}\right) .
$$

Employing (58) in $\bar{f}(u, v)=C_{1}\left(u^{2}+v^{2}\right)^{-1}-A K \mu^{*} \sigma$, we obtain 


$$
\left.f(x, y)=4 C_{1} A^{2}\left[(y+C)^{2}+(x-D)^{2}\right)\right]^{-1}-A K \mu^{*} \sigma .
$$

Substituting for $u(x, y), v(x, y)$ and $f(x, y)$ in equation (3), we have

$$
\begin{aligned}
\vec{H}(x, y)= & \left\{4 C_{1} A^{2}\left[(y+C)^{2}+(x-D)^{2}\right]^{-1}-A K \mu^{*} \sigma\right\} \\
& *\left(-\frac{y+C}{2 A}, \frac{x-D}{2 A}\right) .
\end{aligned}
$$

Using $\omega=\frac{1}{A}, \quad j=-K \mu^{*} \sigma$, equations (58), (59) in (5), (6) and integrating, we determine $e(x, y)$. Using this solution for $e(x, y)$ and (58) in (1), the pressure function is found to be

$$
\begin{aligned}
p(x, y)= & \left(\frac{\rho}{8 A^{2}}-\frac{K^{2}{\mu^{*}}^{3} \sigma^{2}}{4}\right)\left[(y+C)^{2}+(x-D)^{2}\right] \\
& +K \mu^{*^{2}} \sigma C_{1} A \ln \left[(y+C)^{2}+(x-D)^{2}\right]+C_{3}
\end{aligned}
$$

where $C_{3}$ is an arbitrary constant.

CASE (II). In this case, we have $L(u, v)=A u^{2}+B v^{2}+C u+D v+E, \quad \bar{f}(u, v)=\frac{-2 A B K \mu^{\cdot} \cdot \sigma}{A+B}$ and $A \neq \pm B$. Proceeding as in case (i), we obtain

$$
\begin{gathered}
\vec{V}=\left(-\frac{y+C}{2 A}, \frac{x-D}{2 B}\right) \\
\vec{H}=\frac{2 A B K \mu^{*} \sigma}{A+B}\left(\frac{y+C}{2 A}, \frac{D-x}{2 B}\right) \\
p(x, y)=\frac{\rho}{8 A B}\left[(y+C)^{2}+(x-D)^{2}\right] \\
-\frac{K^{2} \mu^{*} \sigma^{2}}{2(A+B)}\left[B(y+C)^{2}+A(x-D)^{2}\right]+\frac{3 \alpha_{1}+2 \alpha_{2}}{8}\left(\frac{A-B}{A B}\right)^{2}+C_{4}
\end{gathered}
$$

where $C_{4}$ is an arbitrary constant.

CASE (III). In this case, $L(u, v)=A\left(u^{2}-v^{2}\right)+C u+D v+E$ and $\bar{f}(u, v)=C_{2}$. Flow variables for this case are:

$$
\begin{gathered}
\vec{V}=\left(-\frac{y+C}{2 A}, \frac{D-x}{2 A}\right) \\
\vec{H}=C_{2} \vec{V} \\
p(x, y)=C_{5}-\frac{\rho}{8 A^{2}}\left[(y+C)^{2}+(x-D)^{2}\right]+\frac{3 \alpha_{1}+2 \alpha_{2}}{2 A^{2}}
\end{gathered}
$$

where $C_{5}$ is an arbitrary constant.

INFINITELY CONDUCTING FLUID. Using the expressions for $L, \bar{J}, \bar{\omega}, W_{1}, W_{2}, F_{1}, F_{2}$ as given by (52), (53), (37) in equations (29) and (36), we find that $\bar{f}(u, v)$ must satisfy

$$
\left.\begin{array}{c}
A u \frac{\partial \bar{f}}{\partial v}-B v \frac{\partial \bar{f}}{\partial u}=0 \\
v \frac{\partial \bar{f}}{\partial u}-u \frac{\partial \bar{f}}{\partial v}=0
\end{array}\right\}
$$

Solving equations (64) for $\bar{f}(u, v)$, we find that $\bar{f}(u, v)=\phi\left(u^{2}+v^{2}\right)$ if $A=B$ and $\bar{f}(u, v)=C_{6}$ if $A \neq B$, where $\phi$ is an arbitrary function of its argument and $C_{6}$ is an arbitrary constant. Therefore, we have the following two cases: 
(i) $L(u, v)=A\left(u^{2}+v^{2}\right)+C u+D v+E, \bar{f}(u, v)=\phi\left(u^{2}+v^{2}\right)$, where $\phi$ is an arbitrary function of its argument.

(ii) $L(u, v)=A u^{2}+B v^{2}+C u+D v+E, \bar{f}(u, v)=C_{6}$ where $C_{6}$ is an arbitrary constant and $B \neq A$.

We now consider these two cases separately.

CASE (I). Without loss of generality, we take $\bar{f}(u, v)=u^{2}+v^{2}$. Using $L(u, v)=A\left(u^{2}+v^{2}\right)+$ $C u+D v+E$ in equations (24), we obtain

$$
\vec{V}=(u, v)=\left(-\frac{y+C}{2 A}, \frac{x-D}{2 A}\right)
$$

and therefore.

$$
f(x, y)=\frac{1}{4 A^{2}}\left[(y+C)^{2}+(x-D)^{2}\right]
$$

Employing (65) and (66) in (9), we obtain

$$
j(x, y)=\frac{1}{2 A^{3}}\left[(y+C)^{2}+(x-D)^{2}\right] .
$$

Using (65) to (67) in equations in the physical plane, we obtain

$$
\begin{aligned}
\vec{H} & =\frac{1}{4 A^{2}}\left[(y+C)^{2}+(x-D)^{2}\right]\left(-\frac{y+C}{2 A}, \frac{x-D}{2 A}\right] \\
p(x, y) & =\left(\frac{\rho}{8 A^{2}}-\frac{\mu^{*}}{96 A^{6}}\right)\left[(y+C)^{2}+(x-D)^{2}\right]+C_{7}
\end{aligned}
$$

where $C_{7}$ is an arbitrary constant.

CASE (II). Using $L(u, v)=A u^{2}+B v^{2}+C u+D v+E$ where $A \neq B$, and $\bar{f}(u, v)=C_{6}$, we obtain

$$
\begin{gathered}
\vec{V}=(u, v)=\left(-\frac{y+C}{2 A}, \frac{x-D}{2 B}\right) \\
j(x, y)=\frac{A+B}{2 A B} C_{6} \\
\vec{H}=C_{6} \vec{V} \\
p(x, y)=\frac{\rho}{8 A B}\left[(y+C)^{2}+(x-D)^{2}\right]+\frac{3 \alpha_{1}+2 \alpha_{2}}{8}\left[\frac{A-B}{A B}\right]^{2} \\
-\frac{A+B}{8 A^{2} B^{2}} \mu^{*} C_{6}^{2}\left[B(y+C)^{2}+A(x-D)^{2}\right]+C_{8}
\end{gathered}
$$

where $C_{8}$ is an arbitrary constant.

Summing up, we have the following theorems:

Theorem III. If $L(u, v)=A u^{2}+B v^{2}+C u+D v+E$ is the Legendre transform of a streamfunction for a steady, plane, aligned flow of an incompressible second-grade fluid of finite electrical conductivity, then the flow in the physical plane is:

(a) a vortex flow given by equations (58) to (51) when $A=B$ in $L(u, v)$.

(b) a flow with hyperbolic streamlines with flow variables given by (63) when $B=-A$ in $L(u, v)$.

(c) a flow given by equations (62) with $\frac{(x-D)^{2}}{4 B}+\frac{(y+C)^{2}}{4 A}=$ constant as the streamlines when $B \neq \pm A$.

TheOREM IV. If $L(u, v)=A u^{2}+B v^{2}+C u+D v+E$ is the Legendre transform of a streamfunction for a steady, plane, aligned, incompressible infinitely conducting second-grade fluid flow, then the flow in the physical plane is: 
(a) a vortex flow with flow variables given by (65)-(68) when $A=B$ in $L(u, v)$.

(b) a flow with flow variables given by equations (69) with $\frac{(x-D)^{2}}{4 B}+\frac{(y+C)^{2}}{4 A}=$ constant as the streamlines when $B \neq A$ in $L(u, v)$.

Application II: We let

$$
L(u, v)=(A u+B) v+C u^{2}+D u+E
$$

to be the Legendre transform function. where $A, B, C, D, E$ are arbitrary constants and $A$ is nonzero.

Evaluating $\bar{J}, \bar{\omega}, W_{1}$ and $W_{2}$, by using (70) in equations (31) to (33), we get

$$
\bar{J}=-\frac{1}{A^{2}}, \quad \bar{\omega}=-\frac{2 C}{A^{2}}, \quad W_{1}=W_{2}=0 .
$$

FINITELY CONDUCTING FLUID. Using equations (28), (70), (71) in equations (29), (30), (35), we find that equation (35) is identically satisfied and $\bar{f}(u, v)$ must satisfy equations

$$
\begin{gathered}
(2 C u+A v) \frac{\partial \bar{f}}{\partial v}-A u \frac{\partial \bar{f}}{\partial u}=0 \\
2 C \bar{f}+(2 C v-A u) \frac{\partial \bar{f}}{\partial v}-A v \frac{\partial \bar{f}}{\partial u}=A^{2} K \mu^{*} \sigma .
\end{gathered}
$$

Multiplying (72) by $v,(73)$ by $u$ and subtracting, we obtain

$$
\frac{\partial \bar{f}}{\partial v}-\frac{2 C u}{A\left(u^{2}+v^{2}\right)} \bar{f}+A K \mu^{*} \sigma \frac{u}{u^{2}+v^{2}}=0
$$

Solving equations (72) and (74), we get

$$
\bar{f}(u, v)=\left\{\begin{array}{cc}
\exp \left[\frac{2 C}{A} \tan ^{-1} \frac{v}{u}\right] \phi(u)+\frac{A^{2}}{2 C} K \mu^{*} \sigma, & C \neq 0 \\
-A K \mu^{*} \sigma \tan ^{-1}\left(\frac{u}{v}\right)+\psi(u), & C=0
\end{array}\right.
$$

where arbitrary functions $\phi(u)$ and $\psi(u)$ must satisfy

$$
\begin{aligned}
{\left[A u \phi^{\prime}(u)\right] v^{2}-[4 C u \phi(u)] v+\left[A u^{3} \phi^{\prime}(u)-\frac{4 C^{2}}{A} u^{2} \phi(u)\right] } & =0, \\
C & \neq 0
\end{aligned}
$$

and

$$
\begin{aligned}
{\left[u \psi^{\prime}(u)\right] v^{2}+\left[2 A K \mu^{*} \sigma u\right] v+\left[u^{3} \psi^{\prime}(u)\right] } & =0, \\
C & =0 .
\end{aligned}
$$

Equations (75) and (76) hold true for all $v$ if $\phi(u)=0$ and $\psi(u)=D_{1}$, where $D_{1}$ is an arbitrary constant. Therefore, we have the following two cases:

(i) $L(u, v)=(A u+B) v+C u^{2}+D u+E, \bar{f}(u, v)=\frac{A^{2}}{2 C} K \mu^{*} \sigma$, when $C \neq 0$.

(ii) $L(u, v)=(A u+B) v+D u+E, \bar{f}(u, v)=D_{1}$.

Using $L(u, v)$ and $\bar{f}(u, v)$ for the two cases and proceeding as in application I, the flow variables in the physical plane are obtained to be: 
CASE (I).

$$
\begin{aligned}
& \vec{V}=(u, v)=\left(\frac{x-B}{A}, \frac{2 C B-A D-2 C x-A y}{A^{2}}\right) \\
& \vec{H}=\frac{A^{2} K \mu^{*} \sigma}{2 C} \vec{V} \\
& p(x, y)=-\frac{\rho}{2 A^{2}}\left[(x-B)^{2}+(y+D)^{2}\right] \\
&-\frac{K^{2} \mu^{*^{3} \sigma^{2}}}{2 C}\left[A y(x-B)+C(x-B)^{2}+A D x\right] \\
&+\left(6 \alpha_{1}+4 \alpha_{2}\right) \frac{A^{2}+C^{2}}{A^{4}}+D_{2}
\end{aligned}
$$

where $D_{2}$ is an arbitrary constant.

CASE (II).

$$
\begin{gathered}
\vec{V}=(u, v)=\left(\frac{x-B}{A},-\frac{y+D}{A}\right) \\
\vec{H}=D_{1} \vec{V} \\
p(x, y)=D_{3}-\frac{\rho}{2 A^{2}}\left[(x-B)^{2}+(y+D)^{2}\right]+\frac{6 \alpha_{1}+4 \alpha_{2}}{A^{2}}
\end{gathered}
$$

where $D_{3}$ is an arbitrary constant.

INFINITELY CONDUCTING FLUID. Using $L, \bar{J}, \bar{\omega}, W_{1}, W_{2}, F_{1}, F_{2}$ given by (70), (71), (37) in equations (29) and (36), we find that $\bar{f}(u, v)$ must satisfy

$$
\left.\begin{array}{rl}
(2 C u+A v) \frac{\partial \bar{f}}{\partial v}-A u \frac{\partial \bar{f}}{\partial u} & =0 \\
v \frac{\partial \bar{f}}{\partial u}-u \frac{\partial \bar{f}}{\partial v} & =0
\end{array}\right\}
$$

Solving (80), we obtain

$$
\bar{f}(u, v)=D_{4}
$$

where $D_{4}$ is an arbitrary constant.

We employ $L(u, v), \bar{f}(u, v)$ given by $(70),(80)$ respectively in (24), (3) and equations in the physical plane, and obtain

$$
\begin{gathered}
\vec{V}=(u, v)=\left(\frac{x-B}{A}, \frac{2 C B-A D-2 C x-A y}{A^{2}}\right) \\
\vec{H}=D_{4} \vec{V}
\end{gathered}
$$

and

$$
\begin{aligned}
p(x, y)=D_{5} & -\frac{\rho}{2 A^{2}}\left[(x-B)^{2}+(y+D)^{2}\right] \\
& -\frac{2 D_{4}^{2} \mu^{*} C}{A^{3}}\left[y(x-B)+\frac{C}{A}(x-B)^{2}+D x\right] \\
& +\left(6 \alpha_{1}+4 \alpha_{2}\right) \frac{A^{2}+C^{2}}{A^{4}}
\end{aligned}
$$

where $D_{5}$ is an arbitrary constant.

Summing up, we have the following theorems:

TheOREM V. If $L(u, v)=(A u+B) v+C u^{2}+D u+E$ is the Legendre transform function of a streamfunction for a steady, plane, aligned, incompressible, finitely conducting second-grade fluid flow, then the flow in the physical plane is 
(a) given by equations (77) having $C x^{2}+A x y-A B y+(A D-2 B C) x=$ constant as its streamlines when $C \neq 0$ in $L(u, v)$.

(b) a flow with rectangular hyperbolas $(x-B)(y+D)=$ constant as its streamlines and is given by equations (78) when $C=0$ in $L(u, v)$.

THEOREM VI. If $L(u, v)=A(u+B) v+C u^{2}+D u+E$ is the Legendre transform function of a streamfunction of a steady, plane, aligned, incompressible, infinitely conducting second-grade fluid flow, then the flow in the physical plane is given by equations (81) with

$$
C x^{2}+A x y-A B y+(A D-2 B C) x=\text { constant }
$$

as its streamlines.

ApPlication III: Let

$$
L^{*}(q, \theta)=F(q) ; \quad F^{\prime}(q) \neq 0, \quad F^{\prime \prime}(q) \neq 0 .
$$

Using (83) in (40) to (43) and (51) to evaluate $J^{*}, \omega^{*}, W_{1}^{*}, W_{2}^{*}, x$ and $y$, we get

$$
\begin{aligned}
J^{*} & =\frac{q}{F^{\prime}(q) F^{\prime \prime}(q)}, & \omega^{*}=\frac{q F^{\prime \prime}(q)+F^{\prime}(q)}{F^{\prime}(q) F^{\prime \prime}(q)}, \\
W_{1}^{*} & =-\frac{1}{q} \omega^{* \prime} \cos \theta F^{\prime}(q), & W_{2}^{*}=\frac{1}{q} \omega^{* \prime} \sin \theta F^{\prime}(q), \\
x & =F^{\prime}(q) \sin \theta, & y=-F^{\prime}(q) \cos \theta
\end{aligned}
$$

We now study finitely conducting fluid flow and infinitely conducting fluid flow as applications of corrolaries I and II respectively.

FINITELY CONLUCTING FLUID. Eliminating $L^{*}, J^{*}, W_{1}^{*}, W_{2}^{*}$ from equations (46), (47) and (48) by using (\$2) and (83), we find that $F(q)$ and $f^{*}(q, \theta)$ must satisfy

$$
\begin{gathered}
f^{*} \omega^{*}+\frac{q}{F^{\prime \prime}(q)} \frac{\partial f^{*}}{\partial q}+K \mu^{*} \sigma=0 \\
\frac{\partial f^{*}}{\partial \theta}=0 \\
\omega^{* \prime}(q)+F^{\prime}(q)\left[\frac{\omega^{* \prime}(q)}{F^{\prime \prime}(q)}\right]^{\prime}=0
\end{gathered}
$$

so that $F(q)$ is the Legendre transform function of a streamfunction. Here $\omega^{*}(q)$ is given in (83) and $j^{*}=-K \mu^{*} \sigma$ has been used for the finitely conducting case. Since equation (86) is identically satisfied when $\omega^{* \prime}=0$ and can be rewritten as

$$
\frac{\omega^{* \prime \prime}(q)}{\omega^{* \prime}(q)}+\frac{F^{\prime \prime}(q)}{F^{\prime}(q)}-\frac{F^{\prime \prime \prime}(q)}{F^{\prime \prime}(q)}=0
$$

when $\omega^{* \prime}(q) \neq 0$, it follows that we have to deal separately with $L^{*}(q, \theta)=F(q)$ having variable vorticity and $L^{*}(q, \theta)=F(q)$ having constant vorticity.

CASE 1. (Variable Vorticity). From the expressions for $x, y$ in (83), we have

$$
r=\sqrt{x^{2}+y^{2}}= \pm F^{\prime}(q), \quad \frac{d r}{d q}= \pm F^{\prime \prime}(q)
$$

Integrating (87) twice with respect to $q$, we obtain

$$
\omega^{*}(q)=M_{1} \ln \left|F^{\prime}(q)\right|+M_{2}
$$


where $M_{1}$ and $M_{2}$ are arbitrary constants.

Substituting for $\omega^{*}(q)$ given in (83) into (89), we get

$$
q+r \frac{d q}{d r}= \pm\left(M_{1} r \ln r+M_{2} r\right)
$$

since $F^{\prime \prime}(q) \neq 0$ and, therefore, $\frac{d r}{d q}=\left(\frac{d q}{d r}\right)^{-1}$.

Integrating the differential equation (90), we get

$$
q= \pm\left[\frac{M_{1}}{2} r \ln r+\left(\frac{2 M_{2}-M_{1}}{4}\right) r+\frac{M_{3}}{r}\right]
$$

where $M_{3}$ is an arbitrary constant.

Employing (88), (91) in equations (51) and making use of the definitions $u=q \cos \theta, v=$ $q \sin \theta$ and $\omega=\frac{\partial v}{\partial x}-\frac{\partial u}{\partial y}$, we obtain

$$
\begin{gathered}
u(x, y)=-y\left[\frac{M_{1}}{4} \ln \left(x^{2}+y^{2}\right)+\left(\frac{2 M_{2}-M_{1}}{4}\right)+\frac{M_{3}}{x^{2}+y^{2}}\right] \\
v(x, y)=x\left[\frac{M_{1}}{4} \ln \left(x^{2}+y^{2}\right)+\left(\frac{2 M_{2}-M_{1}}{4}\right)+\frac{M_{3}}{x^{2}+y^{2}}\right] \\
\omega(x, y)=\frac{M_{1}}{2} \ln \left(x^{2}+y^{2}\right)+M_{2}
\end{gathered}
$$

Using expression for $\omega^{*}(q)$ from (83) in equation (84) and making use of (85), we have

$$
\frac{d}{d q}\left[q F^{\prime}(q) \phi(q)+\frac{K \mu^{*} \sigma}{2} F^{\prime 2}(q)\right]=0, \quad f^{*}(q, \theta)=\phi(q)
$$

Integrating the above equation, we get

$$
f^{*}(q, \theta)=\phi(q)=\frac{M_{4}}{q F^{\prime}(q)}-\frac{K \mu^{*} \sigma F^{\prime}(q)}{2 q}
$$

where $M_{4}$ is an arbitrary constant.

Substituting expressions for $F^{\prime}(q)$ and $q$ given by (88) and (91) respectively into equation (93), we obtain

$$
\begin{aligned}
f(x, y)= & M_{4}\left[\frac{M_{1}}{4}\left(x^{2}+y^{2}\right) \ln \left(x^{2}+y^{2}\right)+\left(\frac{2 M_{2}-M_{1}}{4}\right)\left(x^{2}+y^{2}\right)+M_{3}\right]^{-1} \\
& -\frac{1}{2} K \mu^{*} \sigma \sqrt{x^{2}+y^{2}}\left[\frac{M_{1}}{4} \sqrt{x^{2}+y^{2} \ln \left(x^{2}+y^{2}\right)}\right. \\
& \left.+\left(\frac{2 M_{2}-M_{1}}{4}\right) \sqrt{x^{2}+y^{2}}+\frac{M_{3}}{\sqrt{x^{2}+y^{2}}}\right]^{-1}
\end{aligned}
$$

We use $u(x, y), v(x, y)$ and $f(x, y)$ given by (92) and (94) in equation (3) and obtain

$$
\vec{H}(x, y)=\left(\frac{-M_{4} y}{x^{2}+y^{2}}+\frac{K \mu^{*} \sigma y}{2}, \frac{M_{4} x}{x^{2}+y^{2}}-\frac{K \mu^{*} \sigma x}{2}\right) .
$$


Using $j=-\mu^{*} \sigma K$, equations (92), (94) in equations (5) and (6) and integrating, we get $e(x, y)$. Employing this solution for $e(x, y)$ and (92) in (1), the pressure function is determined to be

$$
\begin{aligned}
p(x, y)= & \frac{\rho M_{1}}{8}\left[M_{3}+\frac{M_{1}}{4}\left(x^{2}+y^{2}\right)\right]\left[\ln \left(x^{2}+y^{2}\right)\right]^{2} \\
& +\frac{\rho}{2}\left[M_{2} M_{3}-\frac{M_{1} M_{3}}{2}+\left(\frac{M_{1} M_{2}-M_{1}^{2}}{4}\right)\left(x^{2}+y^{2}\right)\right] \\
& *\left[\ln \left(x^{2}+y^{2}\right)\right]+\frac{\rho}{4}\left[\frac{M_{2}^{2}}{2}+\frac{5 M_{1}^{2}}{8}-M_{1} M_{2}\right]\left(x^{2}+y^{2}\right) \\
& -\frac{\rho M_{3}}{3}\left(x^{2}+y^{2}\right)^{-1}-\mu M_{1} \tan ^{-1}\left(\frac{x}{y}\right) \\
& +\alpha_{1} M_{1}\left[\frac{M_{1}}{4} \ln \left(x^{2}+y^{2}\right)+\left(\frac{2 M_{2}-M_{1}}{4}\right)+\frac{M_{3}}{x^{2}+y^{2}}\right] \\
& +K \mu^{* 2} \sigma\left[\frac{M_{4}}{2} \ln \left(x^{2}+y^{2}\right)-\frac{K \mu^{*} \sigma}{4}\left(x^{2}+y^{2}\right)\right] \\
& +\frac{3 \alpha_{1}+2 \alpha_{2}}{2}\left[\frac{M_{1}^{2}}{4}+\frac{4 M_{3}^{2}}{\left(x^{2}+y^{2}\right)^{2}} \frac{M_{1} M_{3}}{x^{2}+y^{2}}\right] \\
& +M_{5}
\end{aligned}
$$

where $M_{5}$ is an arbitrary constant.

CASE 2. (Constant Vorticity).

Using $\omega^{*}=\omega_{0}=$ constant in the expression for $\omega^{*}$ given in (83), we have

$$
q F^{\prime \prime}(q)+F^{\prime}(q)-\omega_{0} F^{\prime}(q) F^{\prime \prime}(q)=0 .
$$

Integrating equation (97) with respect to $q$, we get

$$
\omega_{0} F^{\prime 2}(q)-2 q F^{\prime}(q)+2 M_{6}=0
$$

where $M_{6}$ is an arbitrary constant and if $\omega_{0}=0$ then $M_{6} \neq 0$.

Substituting for $F^{\prime}(q)$ given by (88) into equation (98) and solving for $q$, we obtain

$$
q= \pm\left[\frac{\omega_{0}}{2} \sqrt{x^{2}+y^{2}}+\frac{M_{6}}{\sqrt{x^{2}+y^{2}}}\right] .
$$

The solution for $f^{*}(q, \theta)$ satisfying equations (84) and (85) is given by (93), where now $q$ is given by (99).

We proceed as in variable vorticity case and obtain

$$
\begin{gathered}
u(x, y)=-y\left[\frac{\omega_{0}}{2}+\frac{M_{6}}{x^{2}+y^{2}}\right] \\
v(x, y)=x\left[\frac{\omega_{0}}{2}+\frac{M_{6}}{x^{2}+y^{2}}\right] \\
f(x, y)=M_{4}\left[\frac{\omega_{0}}{2}\left(x^{2}+y^{2}\right)+M_{6}\right]^{-1} \\
K \mu^{*} \sigma-\frac{x^{2}+y^{2}}{\omega_{0}\left(x^{2}+y^{2}\right)+2 M_{6}} \\
\vec{H}(x, y)=\left(-\frac{M_{4} y}{x^{2}+y^{2}}+\frac{k \mu^{*} \sigma y}{2}, \frac{M_{4} x}{x^{2}+y^{2}}-\frac{K \mu^{*} \sigma x}{2}\right)
\end{gathered}
$$


and

$$
\begin{aligned}
p(x, y)= & \rho\left[\frac{\omega_{0}^{2}}{8}\left(x^{2}+y^{2}\right)+\frac{\omega_{0} M_{6}}{2} \ln \left(x^{2}+y^{2}\right)-\frac{M_{6}^{2}}{2\left(x^{2}+y^{2}\right)}-\frac{\omega_{0} M_{6}}{2}\right] \\
& K \mu^{* 2} \sigma\left[\frac{M_{4}}{2} \ln \left(x^{2}+y^{2}\right)-\frac{K \mu^{*} \sigma}{4}\left(x^{2}+y^{2}\right)\right] \frac{\left.6 \alpha_{1}+4 \alpha_{2}\right) M_{6}^{2}}{\left(x^{2}+y^{2}\right)^{2}}+M_{\tau}
\end{aligned}
$$

where $M_{7}$ is an arbitrary constant.

INFINITELY CONDUCTING FLUID. Employing the expressions for $L^{*}, J^{*}, W_{1}^{*}, W_{2}^{*}, F_{1}^{*}$ and $F_{2}^{*}$ as given by (83), (84), (44) and (45) in equations (47), (49), we find that $F(q)$ and $f^{*}(q, \theta)$ must satisfy

$$
\begin{gathered}
\frac{\partial f^{*}}{\partial \theta}=0 \\
\mu\left\{\omega^{* \prime}+F^{\prime}\left[\frac{\omega^{* \prime}}{F^{\prime \prime}}\right]^{\prime}\right\}-2 \mu^{*} f^{*} \frac{\partial f^{*}}{\partial \theta}=0 .
\end{gathered}
$$

Solving (101), (102), we obtain

$$
f^{*}(q, \theta)=\phi(q)
$$

where $\phi$ is an arbitrary function of its argument, and $F(q)$ satisfying

$$
\omega^{* \prime}+F^{\prime}\left[\frac{\omega^{* \prime}}{F^{\prime \prime}}\right]^{\prime}=0
$$

Analysing equation (104), we have the following two cases as in finitely conducting fluid flow:

(1) $L^{*}(q, \theta)=F(q)$ having variable vorticity.

(2) $L^{*}(q, \theta)=F(q)$ having constant vorticity.

CASE 1. (Variable Vorticity). Following the same analysis as in finitely conducting fluid flow, we obtain $q, u(x, y), v(x, y), \omega(x, y)$ as given by (91) and (92). Hence,

$$
f(x, y)=\phi(q)
$$

where $q$ is given by (91).

Proceeding as in previous example, we get

$$
\begin{aligned}
j(x, y)= & \phi(q)\left[\frac{M_{1}}{2} \ln \left(x^{2}+y^{2}\right)+M_{2}\right] \\
& +\phi^{\prime}(q)\left[\frac{M_{1}}{4} \ln \left(x^{2}+y^{2}\right)+\left(\frac{2 M_{2}-M_{1}}{4}\right)\right. \\
& \left.+\frac{M_{3}}{x^{2}+y^{2}}\right]\left[\frac{M_{1}}{4} \sqrt{x^{2}+y^{2}} \ln \left(x^{2}+y^{2}\right)\right. \\
& \left.+\left(\frac{2 M_{2}+M_{1}}{4}\right) \sqrt{x^{2}+y^{2}}-\frac{M_{3}}{\sqrt{x^{2}+y^{2}}}\right] \\
& \vec{H}(x, y)=\phi(q)(u(x, y), v(x, y))
\end{aligned}
$$




$$
\begin{aligned}
p(x, y)= & \frac{\rho}{8} M_{1}\left[M_{3}+\frac{M_{1}}{4}\left(x^{2}+y^{2}\right)\right]\left[\ln \left(x^{2}+y^{2}\right)\right]^{2} \\
& +\frac{\rho}{2}\left[M_{2} M_{3}-\frac{M_{1} M_{3}}{2}+\left(\frac{M_{1} M_{2}-M_{1}^{2}}{4}\right)\left(x^{2}+y^{2}\right)\right] \\
& *\left[\ln \left(x^{2}+y^{2}\right)\right]+\frac{\rho}{4}\left[\frac{M_{2}^{2}}{2}+\frac{5 M_{1}^{2}}{8}-M_{1} M_{2}\right]\left(x^{2}+y^{2}\right) \\
& -\frac{\rho M_{3}}{2}\left(x^{2}+y^{2}\right)^{-1}-\mu M_{1} \tan ^{-1}\left(\frac{x}{y}\right) \\
& +\alpha_{1} M_{1}\left[\frac{M_{1}}{4} \ln \left(x^{2}+y^{2}\right)+\left(\frac{2 M_{2}-M_{1}}{4}\right)+\frac{M_{3}}{x^{2}+y^{2}}\right] \\
& +\mu^{*}\left[\int j H_{2} d x+\int j H_{1} d y\right] \\
& +\frac{3 \alpha_{1}+2 \alpha_{2}}{2}\left[\frac{M_{1}^{2}}{4}+\frac{4 M_{3}^{2}}{\left(x^{2}+y^{2}\right)^{2}}-\frac{M_{1} M_{3}}{x^{2}+y^{2}}\right]+M_{8}
\end{aligned}
$$

where $M_{8}$ is an arbitrary constant and $j, H_{1}, H_{2}$ are given by (106), (107).

CASE 2. (Constant Vorticity). In this case, using $L^{*}(q, \theta)=F(q)$ having constant vorticity $\omega^{*}=$ $\omega_{0}$, and $f^{*}(q, \theta)=\phi(q)$, we obtain $q, u(x, y), v(x, y)$ given by (99) and in $(100), f(x, y)=\phi(q)$ where $q$ is now given by (99),

$$
\begin{aligned}
& j(x, y)= \omega_{0} \phi(q)+\phi^{\prime}(q)\left[\frac{\omega_{0}}{2}+\frac{M_{6}}{x^{2}+y^{2}}\right] *\left[\frac{\omega_{0}}{2} \sqrt{x^{2}+y^{2}}-\frac{M_{6}}{\sqrt{x^{2}+y^{2}}}\right] \\
& \vec{H}(x, y)=\phi(q)(u(x, y), v(x, y)) \\
& p(x, y)=\rho\left[\frac{\omega_{0}^{2}}{8}\left(x^{2}+y^{2}\right)+\frac{\omega_{0} M_{6}}{2} \ln \left(x^{2}+y^{2}\right)-\frac{M_{6}^{2}}{2\left(x^{2}+y^{2}\right)}-\frac{\omega_{0} M_{6}}{2}\right] \\
&+\mu^{*}\left[\int j H_{2} d x+\int j H_{1} d y\right]+\frac{\left.6 \alpha_{1}+4 \alpha_{2}\right) M_{6}^{2}}{\left(x^{2}+y^{2}\right)^{2}}+M_{9}
\end{aligned}
$$

where $M_{9}$ is an arbitrary constant, and $j, H_{1}, H_{2}$ are given by (109), (110).

Summing up, we have the following theorems:

THEOREM VII. If $L^{*}(q, \theta)=F(q)$ is the Legendre transform function of a streamfunction for a steady. plane, aligned, incompressible, finitely conducting second-grade fluid flow, then the flow in the physical plane is

(a) given by equations (92), (94) to (96) with $\left(x^{2}+y^{2}\right)\left[\frac{M_{1}}{8} \ln \left(x^{2}+y^{2}\right)+\frac{M_{2}-M_{1}}{4}\right]+$ $\frac{M_{3}}{2} \ln \left(x^{2}+y^{2}\right)=$ constant as its streamlines, when vorticity is not a constant.

(b) given by equations (100) having $\omega_{0}\left(x^{2}+y^{2}\right)+2 M_{6} \ell n\left(x^{2}+y^{2}\right)=$ constant as its streamlines, when vorticity is a constant.

THEOREM VIII. If $L^{*}(q, \theta)=F(q)$ is the Legendre transform function of a streamfunction for a steady. plane, aligned, incompressible, infinitely conducting second grade fluid flow, the flow in the physical plane is

(a) given by equations (92), (105) to (108) having $\left(x^{2}+y^{2}\right)\left[\frac{M_{1}}{8} \ln \left(x^{2}+y^{2}\right)+\frac{M_{2}-M_{1}}{4}\right]+$ $\frac{M_{3}}{2} \ln \left(x^{2}+y^{2}\right)=$ constant as its streamlines, when vorticity is not a constant.

(b) given by equations (100), (109) to (111) with $\omega_{0}\left(x^{2}+y^{2}\right)+2 M_{6} \ln \left(x^{2}+y^{2}\right)=$ constant as its streamlines, when vorticity is a constant. 
APPLICATION IV. Let

$$
L^{*}(q, \theta)=A \theta+B
$$

be the Legendre transform function, where $A, B$ are arbitrary constants and $A$ is nonzero.

We evaluate $J^{*}, \omega^{*}, W_{1}^{*}, W_{2}^{*}$ by using (112) in equations (40) to (43) and obtain

$$
J^{*}=-\frac{q^{4}}{A^{2}}, \quad \omega^{*}=W_{1}^{*}=W_{2}^{*}=0 .
$$

FINITELY CONDUCTING FLUID. Employing (112), (113) in equations (46), (47) and (48), we find that equation $(48)$ is identically satisfied and $f^{*}(q, \theta)$ must satisfy

$$
\left.\begin{array}{l}
\frac{\partial f^{*}}{\partial \theta}=-\frac{A K \mu^{*} \sigma}{q^{2}} \\
\frac{\partial f^{*}}{\partial q}=0
\end{array}\right\}
$$

Solving equations (114), we obtain

$$
f^{*}(q, \theta)=\phi(\theta)
$$

where an arbitrary function $\phi(\theta)$ must satisfy

$$
q^{2} \phi^{\prime}(\theta)+A \mu^{*} \sigma K=0 .
$$

Equation (115) holds true for all $q$ if $\phi^{\prime}(\theta)=0$ and $A K \mu^{*} \sigma=0$. Therefore, we have

$$
f^{*}=(q, \theta)=\phi(\theta)=N_{1}
$$

where $N_{1}$ is an arbitrary constant and $K=0$.

Using $L^{*}(q, \theta)=A \theta+B$ and $f^{*}(q, \theta)=N_{1}$, we obtain

$$
\begin{gathered}
\vec{V}=(u, v)=\left(\frac{A x}{x^{2}+y^{2}}, \frac{A y}{x^{2}+y^{2}}\right) \\
j=0, \vec{H}=N_{1} \vec{V} \\
p(x, y)=N_{2}-\frac{\rho A^{2}}{2\left(x^{2}+y^{2}\right)}+\left(6 \alpha_{1}+4 \alpha_{2}\right)\left(\frac{A}{x^{2}+y^{2}}\right)^{2}
\end{gathered}
$$

where $N_{2}$ is an arbitrary constant.

INFINITELY CONDUCTING FLUID. Employing $L^{*}, J^{*}, \omega^{*}, W_{1}^{*}, W_{2}^{*}, F_{1}^{*}$ and $F_{2}^{*}$ given by (112), (113), (44) and (45) in equations (47), (49), we find that $f^{*}(q, \theta)$ must satisfy

$$
\left.\begin{array}{l}
\frac{\partial f^{*}}{\partial q}=0 \\
\frac{\partial f^{*}}{\partial \theta}=0
\end{array}\right\}
$$

Solving equations (118), we obtain

$$
f^{*}(q, \theta)=N_{3}
$$

where $N_{3}$ is an arbitrary constant.

Proceeding as before, we have the following results:

$$
\begin{gathered}
\vec{V}=(u, v)=\left(\frac{A x}{x^{2}+y^{2}}, \frac{A y}{x^{2}+y^{2}}\right) \\
j=0 \\
\vec{H}(x, y)=N_{3} \vec{V}
\end{gathered}
$$




$$
p(x, y)=N_{4}-\frac{\rho A^{2}}{2\left(x^{2}+y^{2}\right)}+\left(6 \alpha_{1}+4 \alpha_{2}\right)\left(\frac{A}{x^{2}+y^{2}}\right)^{2}
$$

where $N_{\mathbf{4}}$ is an arbitrary constant.

Summing up, we have the following theorem.

THEOREM IX. If $L^{*}(q, \theta)=A \theta+B$ is the Legendre transform function of a streamfunction for a steady, plane, aligned, incompressible, finitely conducting second-grade fluid flow, then the flow in the physical plane is given by equations (117) with $\tan ^{-1}\left(\frac{x}{y}\right)=$ constant as its streamlines.

THEOREM X. If $L^{*}(q, \theta)=A \theta+B$ is the Legendre transform function of a streamfunction for a steady, plane, aligned, incompressible, infinitely conducting second-grade fluid flow, then the flow in the physical plane is given by equations (119) having $\tan ^{-1}\left(\frac{x}{y}\right)=$ constant as its streamlines.

\section{REFERENCES}

1. W. F. Ames, "Non-linear partial differential equations," Academic Press, New York, 1965.

2. A. M. Siddiqui, P. N. Kaloni and O. P. Chandna, Hodograph transformation methods in non-Newtonian fluids, J. of Engineering Mathenatics 19 (1986), 203-216.

3. O. P. Chandna, R. M. Barron and A. C. Smith, Rotational plane flows of a viscous flurd, Siam J. Appl. Math. 42 (1982), 1323-1336.

4. M. H. Martin, The flow of a viscous fluid I, Arch. Rat. Mech. Anal. 41 (1971), 266-286. 


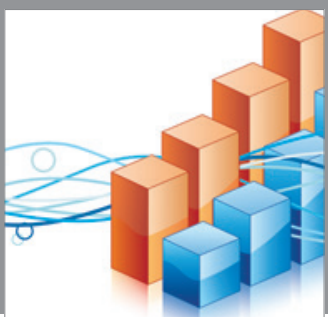

Advances in

Operations Research

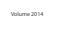

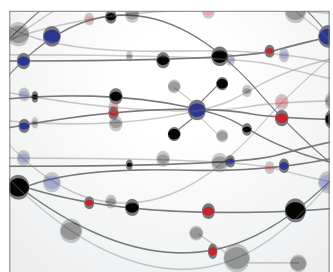

\section{The Scientific} World Journal
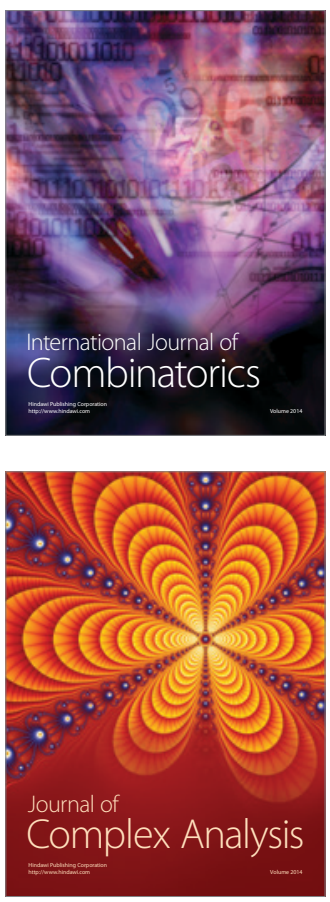

International Journal of

Mathematics and

Mathematical

Sciences
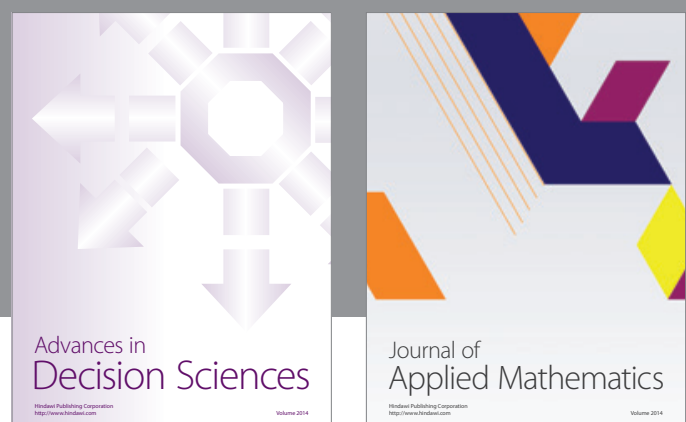

Journal of

Applied Mathematics
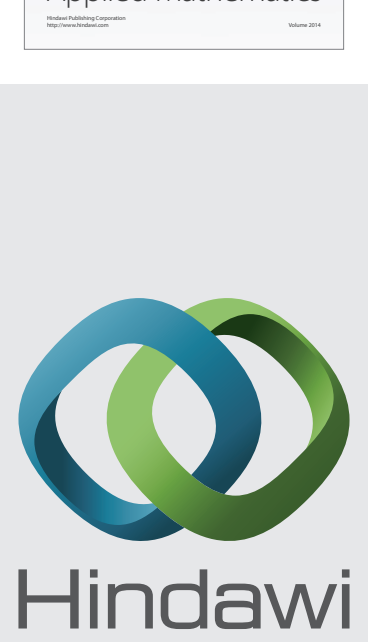

Submit your manuscripts at http://www.hindawi.com
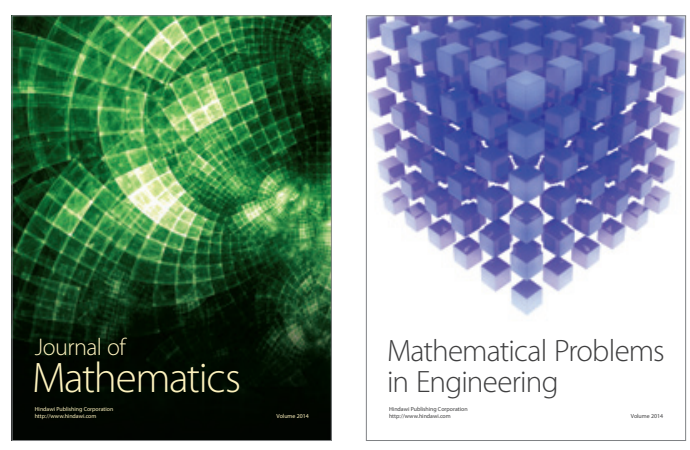

Mathematical Problems in Engineering
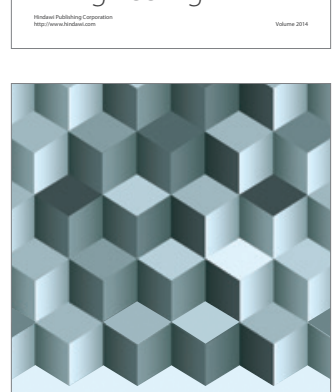

Journal of

Function Spaces
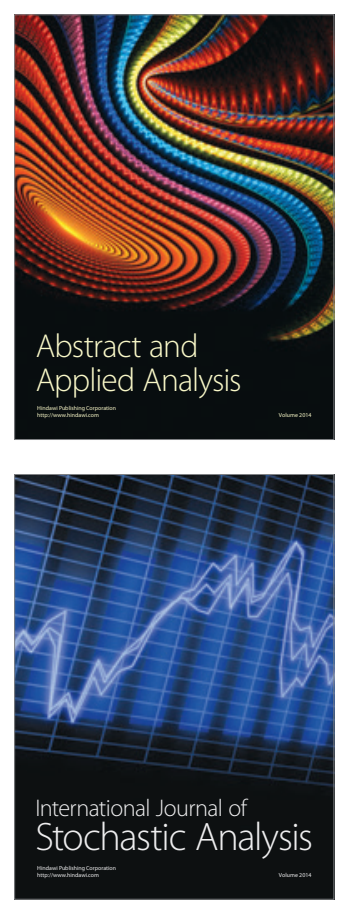

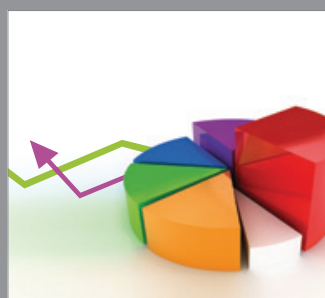

ournal of

Probability and Statistics

Promensencen
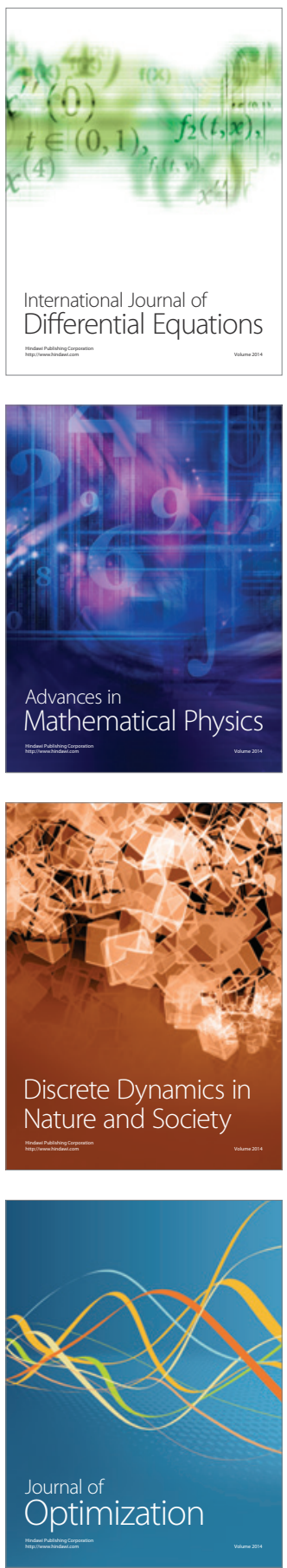